Meta

Journal des traducteurs

Translators' Journal

\title{
Affiche, enseigne, étiquette, pancarte, panneau ou plaque?
}

\section{Jean-Marc Lambert}

Volume 24, numéro 4, décembre 1979

URI : https://id.erudit.org/iderudit/003382ar

DOI : https://doi.org/10.7202/003382ar

Aller au sommaire du numéro

Éditeur(s)

Les Presses de l'Université de Montréal

ISSN

0026-0452 (imprimé)

1492-1421 (numérique)

Découvrir la revue

Citer cet article

Lambert, J-M. (1979). Affiche, enseigne, étiquette, pancarte, panneau ou plaque? Meta, 24(4), 464-465. https://doi.org/10.7202/003382ar d'utilisation que vous pouvez consulter en ligne.

https://apropos.erudit.org/fr/usagers/politique-dutilisation/ 


\section{AFFICHE, ENSEIGNE, ÉTIQUETTE, PANCARTE, PANNEAU OU PLAQUE?}

Comment faire la distinction entre une affiche, une enseigne, une étiquette, une pancarte, un panneau et une plaque? Les quelques définitions qui suivent devraient permettre d'y voir clair.

L'AFFICHE est généralement une feuille imprimée, en papier souple et de format assez grand. On s'en sert pour transmettre des conseils de prudence affiches de sécurité, pour annoncer des films, des spectacles, etc., ou pour diffuser des règlements.

L'ENSEIGNE permet de repérer et d'identifier un établissement commercial. Elle est fabriquée en bois, en métal, en plastique ou en tout autre matériau et elle peut être lumineuse. L'enseigne porte le nom ou le symbole de l'établissement. On se souvient de l'air connu : « $\ddot{A}$ l'enseigne de confiance ESSO, à l'enseigne des bons voyages. 》

L'ÉTIQUETTE est essentiellement un petit morceau de papier, de carton, etc. qui porte une inscription courte et précise et qui est fixé à un objet pour en identifier la nature, le contenu, le prix, la destination ou le possesseur. Ainsi, dans les magasins, les articles en vente portent une étiquette de prix. On utilise également des étiquettes pour adresser des lettres, des périodiques ou des colis.

La PANCARTE est un écriteau en bois, en carton ou en métal qui porte un avis, un message ou une inscription. La pancarte est généralement assez rigide et on peut l'apposer sur un mur, un panneau, ou la porter au bout d'un bâton comme on le fait dans des assemblées ou des manifestations.

L'Hydro-Québec utilise des pancartes d'interdiction de monter sur les supports de lignes. Ces pancartes servent à mettre le public en garde contre les risques d'électrocution par contact avec les pièces sous tension et contre les risques de chute pouvant résulter de l'ascension des supports de lignes de transport.

Le PANNEAU est une surface plane (en bois, en métal ou en toile tendue) destinée à servir de support à des inscriptions. Le panneau a généralement des dimensions plus grandes que celles de la pancarte. Lorsqu'il porte des indications le long des routes, on le nomme panneau de signalisation. Lorsqu'il porte un message publicitaire, c'est un panneau-réclame.

Une PLAQUE est une feuille d'une matière rigide, plate et peu épaisse. Comme exemples très connus, on peut mentionner la plaque d'immatriculation d'une automobile et la plaque signalétique d'une machine ou d'un appareil.

JEAN-MARC LAMBerT 


\section{BIBLIOGRAPHIE}

ASSOCIATION FRANÇAISE DE NORMALISATION, Norme française, Présentation des affiches horaires et touristiques - Règles générales et exemples de dispositions, NF F 59-001, Paris, AFNOR, 1949, 3 p.

CHAMINADE, J., 700 mots courants de la publicité et de l'imprimerie, Paris, Eyrolles, 1969, $159 \mathrm{p}$.

Grand Larousse encyclopédique, Paris, Larousse, 1960-1968, 10 vol. et supplément.

QUÉBEC, Ministère de la Voirie, Instruction générale sur la signalisation routière du Québec, Québec, 1966, 225 p.

ROBERT, Paul, Dictionnaire alphabétique et analogique de la langue française, Paris, Littré, 1958-1964, 6 vol. et supplément.

Vigilance, Voici les 40 nouveaux panneaux officiels de signalisation », Paris, Electricité de France, no 33 , s.d., p. 20-22. 